\title{
Die österreichische Protestarena im neuen Jahrtausend: Mobilisierungsstärke, Aktionsformen und Themen, 1998-2016
}

Martin Dolezal

Universität Salzburg / Institut für Höhere Studien (IHS)

martin.dolezal@sbg.ac.at

\section{Zusammenfassung}

Politischer Protest ist auch in Österreich ein normaler Bestandteil der politischen Auseinandersetzung geworden und hat im neuen Jahrtausend seinen vormals „unkonventionellen“ Charakter endgültig verloren. Basierend auf der Berichterstattung der Nachrichtenagentur APA analysiert der Beitrag alle Protestereignisse, die in den Jahren von I998 bis 2016 stattgefunden haben, und konzentriert sich dabei auf drei Charakteristika der österreichischen Protestarena: das Ausmaß der Mobilisierung, die gewählten Aktionsformen und die bei den Protesten angesprochenen Themen. Die Ergebnisse der Protestereignisanalyse zeigen, dass das im Jahr 2000 aufgrund der Bildung der ÖVP-FPÖ-Regierung erreichte Mobilisierungsniveau ein Ausreißer geblieben ist, die Proteste überwiegend moderate Aktionsformen aufweisen und der Umweltschutz bereits vor den aktuellen Klimaschutzprotesten das wichtigste Thema war.

\section{Schlüsselwörter}

Protest, Partizipation, Demokratie, Konflikt, Österreich

\section{The Austrian Protest Arena in the New Millennium: Extent of Mobilization, Forms of Action, and Issues, 1998-2016}

\section{Abstract}

Political protest has become a normal feature of political conflict in Austria as well and has finally lost its formerly ,unconventional" character in the new millennium. Based on the reporting of the news agency APA, the article analyses all protest events that took place in the years from 1998 to 2016 and focusses on three characteristics of the Austrian protest arena: the extent of mobilization, the chosen forms of action, and the issues addressed at the protests. The results of the protest event analysis indicate that the level of mobilization reached in 2000 due to the formation of the ÖVP-FPÖ government has remained an outlier, that the protests predominantly feature moderate forms of action, and that environmental protection was the most important issue even before the current wave of climate protests.

\section{Keywords}

protest, participation, democracy, conflict, Austria

The author has declared that no competing interests exist. 


\section{Einleitung}

Die Beschäftigung mit politischem Protest hat in den vergangenen Jahren in der Politikwissenschaft stark an Bedeutung gewonnen (Rucht 2007). Mehrere große Protestwellen nach der Jahrtausendwende, etwa die Mobilisierung gegen den Irakkrieg (Walgrave/Rucht 20IO), der Widerstand gegen Sparmaßnahmen in Folge der Großen Rezession (Giugni/Grasso 2015; Kriesi et al. 2020) und zuletzt die Klimaschutzproteste der Fridays for Future-Bewegung (de Moor et al. 2020), haben das wissenschaftliche Interesse neu belebt. Wurden Proteste in der klassischen, stark institutionenbezogenen Politikwissenschaft noch als abweichendes, die demokratische Ordnung gefährdendes Verhalten interpretiert, orientiert sich die Forschung spätestens seit der Jahrtausendwende an einem Paradigma der "Normalisierung“. Tatsächlich sind die sozialstrukturellen und ideologischen Unterschiede zwischen in der Protestarena aktiven und inaktiven Personen kleiner geworden (van Aelst/Walgrave 200I). Eine sehr weitgehende These bezeichnet westliche Gesellschaften aufgrund der Alltäglichkeit von Protesten sogar als „Movement Societies“ (Meyer/ Tarrow I998; vgl. auch Neidhardt/Rucht I993).

Österreich galt in Bezug auf das Ausmaß von politischem Protest und die damit verbundenen „unkonventionellen“ Partizipationsformen lange Zeit als exemplarisches Beispiel einer wenig mobilisierten Gesellschaft (z. B. Plasser/Seeber 20I7). Ein starker Staat, dem auch die etablierten Parteien und die in die Sozialpartnerschaft eingebundenen traditionellen Interessenorganisationen zugerechnet werden, dominiert - so die klassische Interpretation - eine schwache Zivilgesellschaft. Ein näherer Blick auf die häufig nur spärlich vorhandenen Daten kann diese traditionelle Interpretation jedoch nur teilweise bestätigen (Dolezal/Hutter 2007; Dolezal 20I9a). Und spätestens die Proteste gegen die Bildung der ÖVP-FPÖ-Koalition im Februar 2000 zeigten, dass auch in Österreich politische Konflikte jenseits von institutionalisierten Kanälen ausgetragen werden. Der vorliegende Beitrag gibt einen Überblick über die Entwicklung der österreichischen Protestlandschaft von I998 bis 20I6. Untersucht werden die Mobilisierungsstärke, die Aktionsformen und die Themen der Proteste.

Als theoretischer Hintergrund dient der die politikwissenschaftliche Protestforschung dominierende Politische Prozess-Ansatz (vgl. Kriesi 2004). Der politische Kontext, so die dem Ansatz zugrundeliegende These, beeinflusst nicht nur das Ausmaß, sondern auch den Charakter der Proteste. Inwieweit haben die generellen Veränderungen der österreichischen Politik die Protestarena beeinflusst? Widerspiegeln die Proteste die Entwicklungen in der Wahlarena, oder bildet die Protestarena eine davon losgelöste Sphäre? Studien zur Entwicklung der österreichischen Politik, etwa zum häufig konstatierten Wandel von einer Konsens- zu einer Konfliktdemokratie (Plasser/Ulram 2002), haben die Protestpolitik nur selten berücksichtigt. Standardwerke zum politischen System übergehen den hier untersuchten Bereich (Dachs et al. 2006) I $^{\mathrm{I}}$ oder widmen ihm nur sehr wenig Platz (Helms/Wineroither 20I2a). Der Großteil der vorhandenen Literatur zur Protestpolitik analysiert nicht deren generelle Entwicklung, sondern beschränkt sich auf markante Episoden, wie die AntiGMO-Bewegung (z. B. Seifert 2009), die unibrenntKampagne(z. B. Brantner/Schwarzenegger 20I2; Maireder/Schwarzenegger 20I2) oder Anti-Asyl-Proteste (z. B. Haselbacher/Rosenberger 20I8).

Die nachfolgende Analyse der österreichischen Protestarena beruht auf Meldungen der Nachrichtenagentur APA (Austria Presse Agentur). ${ }^{2}$ Mit Hilfe einer Protestereignisanalyse (Koopmans/Rucht 2002), einer Form der quantitativen Inhaltsanalyse, wurden zentrale Charakteristika der identifizierten Proteste systematisch erfasst. Eine ähnlich umfassende Analyse, jedoch auf der Basis von Zeitungsartikeln, wurde für Österreich zuvor erst einmal durchgeführt (Dolezal/Hutter 2007).

Nach einer Diskussion der relevanten Merkmale des politischen Kontexts seit der Jahrtausendwende werden Erwartungen für die in diesem Beitrag untersuchten drei Charakteristika der Protestarena formuliert: die Mobilisierungsstärke, die Aktionsformen und die Themen. Anschließend wird auf die Methodik der Datensammlung eingegangen, ehe die zentralen Ergebnisse der Protestereignisanalyse präsentiert werden. Deren Resultate zeigen, dass das im Jahr 2000 aufgrund der Bildung der ÖVP-FPÖ-Regierung erreichte Mobilisierungsniveau ein Ausreißer geblieben ist, die Proteste überwiegend moderate Aktionsformen aufweisen und der Umweltschutz - bereits vor den aktuellen Klimaschutzprotesten das wichtigste Thema der österreichischen Protestarena war.

\section{Politischer Kontext: Die Verfestigung einer Konfliktdemokratie?}

Proteste finden in einem spezifischen politischen Kontext statt, weshalb der Politische Prozess-Ansatz (vgl. u. a. Eisinger I973; McAdam I982; Tarrow 1998) die politikwissenschaftliche Forschung dominiert. Kriesi (2004) unterscheidet in seiner häufig genutzten Konzeptualisierung dieses Ansatzes drei Bereiche: die politischen Opportunitätsstrukturen im engeren Sinn, die Akteurskonfiguration und den Interaktionskontext. Da die

I Der Vorgänger dieses Standardwerks enthielt ein einschlägiges Kapitel (Gottweis I997).

2 Die Sammlung der Daten erfolgte im Rahmen des vom Wissenschaftsfonds FWF geförderten Projekts „Die österreichische Protestarena im 2I. Jahrhundert" (P28I8O-G22). 
vorliegende Untersuchung nicht spezifischen Episoden, sondern der generellen Entwicklung der Protestarena gewidmet ist, sind vor allem die beiden ersten Aspekte von Bedeutung. Unter die politischen Opportunitätsstrukturen fallen bei Kriesi die formalen institutionellen Strukturen des politischen Systems, informelle Verfahren im Umgang mit HerausforderInnen und die zentralen politischen Konfliktlinien. Im Zentrum der Akteurskonfiguration steht die Bedeutung parteipolitischer bzw. institutionalisierter AllianzpartnerInnen. Die Frage ist, welche Ausformungen diese Kontextfaktoren seit der Jahrtausendwende haben und welche Konsequenzen dies für die Protestarena hat.

\subsection{Politische Opportunitätsstrukturen}

Die formalen institutionellen Strukturen beziehen sich vor allem auf das Ausmaß der Offenheit des Zugangs zu Entscheidungsverfahren und der Umsetzungsmöglichkeit staatlicher Entscheidungen. In Summe kann Österreich in der darauf aufbauenden Differenzierung zwischen starken und schwachen Staaten (Badie/Birnbaum I979) der ersten Gruppe zugeordnet werden (Kriesi et al. 1995, 37). Für diese Zuordnung sprechen etwa der, verglichen mit anderen Bundesstaaten, relativ hohe Grad an Zentralisierung (Lijphart 2012, 178) und die Dominanz der Exekutive über die Legislative (Helms/ Wineroither 2012b). Österreich stellt jedoch keinen extremen Fall dar, da etwa der Zugang zum Parlament aufgrund des Verhältniswahlrechts und der niedrigen Mandatsschwelle relativ einfach ist. HerausforderInnen können auch direktdemokratische Instrumente nutzen, doch haben die häufig durchgeführten Volksbegehren einen rein appellativen Charakter. Andere Verfahren können auf Bundesebene von der Bevölkerung nicht initiiert werden. Zudem ist die direktdemokratische Arena sowohl im Bund (Rosenberger/Seeber 2007) als auch in den Ländern (Dolezal 2020) stark von Parteien bzw. Regierungen, d. h. von etablierten AkteurInnen, geprägt.

Informelle Verfahren im Umgang mit HerausforderInnen variieren in einer vergleichenden Perspektive zwischen Strategien des Ausschlusses und der Integration (Kriesi et al. 1995, 33- 37). Sie sind Teil der politischen Kultur eines Landes und daher ein zumindest mittelfristig stabiles Merkmal. Die klassische Einstufung Österreichs als Konkordanz- bzw. Konsensdemokratie (Lehmbruch 1967) spricht für eine dominante Strategie der Integration im Umgang mit politischen HerausforderInnen. Tatsächlich wird Österreich in der vergleichenden Bewegungsforschung als Beispiel von „informal inclusion“ (Kriesi et al. 1995, 37) charakterisiert. Während das System der Sozialpartnerschaft auf die Integration ökonomischer Interessen von Arbeit und Kapital sowie der Landwirtschaft ausgerichtet ist, war die Reaktion des Staates etwa auf die Umweltbewegung zunächst von Konflikten geprägt. Bei neuen, mit der Öffnung von Nationalstaaten verbundenen Themen wie der Migration ist der Umgang mit HerausforderInnen widersprüchlich. Während etwa religiöse Forderungen von MigrantInnen auch dank institutionalisierter Kanäle der Interessenvermittlung lange Zeit auf Akzeptanz stießen, sind politische Beteiligungsrechte immer noch eng mit der StaatsbürgerInnenschaft verbunden (Rosenberger 2OI2).

Im Hinblick auf den dritten Faktor, die zentralen politischen Konfliktlinien (cleavages), kann zunächst auf den unterschiedlich großen Bedeutungsverlust der drei traditionellen Antagonismen verwiesen werden (z. B. Plasser et al. 2000, 56-59): Ökonomische Gegensätze gelten immer noch als zentral, wenngleich sie aufgrund der Einbettung in die Sozialpartnerschaft als befriedet gelten. Der Gegensatz zwischen Kirche und Staat hat seit den I970er Jahren stark an Bedeutung verloren; zuletzt zeigte er sich vor allem in Debatten über christliche Symbole in öffentlichen Gebäuden sowie bei bioethischen Fragen. Auch in Österreich kam es zu einer Transformation der kulturellen Konfliktlinie in einen Gegensatz zwischen libertären und autoritären Werten (Dolezal 2019b). Die nationale Konfliktlinie, der Streit um die Eigenstaatlichkeit bzw. Nationswerdung Österreichs, ist für die Masse der Bevölkerung bedeutungslos geworden (Bruckmüller 1996, 35-85).

Neue, mit der ökonomischen, kulturellen und politischen Öffnung von Nationalstaaten verbundene Konflikte (Kriesi et al. 2008; 2012) weisen im Falle Österreichs markante Unterschiede auf. Dies zeigt auch das unterschiedliche Ausmaß der themenspezifischen Forschung zu politischem Protest:

Ein geringes Konfliktniveau kennzeichnet die ökonomische Dimension der Globalisierung. Auch von den mit der Weltwirtschaftskrise von 2007 bis 2009 (Große Rezession) und der Euro-Krise verbundenen Verwerfungen war Österreich in einem nur relativ geringen Ausmaß betroffen, weshalb diese Krisen deutlich weniger politische Auswirkungen hatten als in anderen Ländern (Luther 2015). Auch abseits der institutionalisierten Politik blieb das Konfliktniveau niedrig: Die Global Justice Movement war in Österreich ein insgesamt peripheres Phänomen (z. B. Boyle 20II), doch spielten bei der im Ländervergleich starken Mobilisierung gegen das Freihandelsabkommen TTIP (Caiani/Graziano 2018) auch Proteste eine relevante Rolle (Rone 2020).

Im Gegensatz dazu war Österreich besonders stark von der Flüchtlings- und Migrationskrise der Jahre 2015/2016 betroffen (Gruber 2017). Der seit den I990er Jahren andauernde Streit um die Zuwanderung (Meyer/Rosenberger 2015) wurde aus Sicht der Bevölkerung zum ersten Mal für längere Zeit das wichtigste politische Thema und hatte auch starke Auswirkungen auf das 
Wahlverhalten (Plasser/Sommer 2018). Migration und die eng damit verwobenen Themen Islam und Rechtspopulismus bzw. Rechtsextremismus waren daher auch Gegenstand von Untersuchungen der Protestpolitik (z. B. Ajanovic et al. 2016; Atac 2016; Berntzen/Weisskircher 2016; Hadj Abdou/Rosenberger 2019a; 2OI9b; Hafez 2016; Haselbacher/Rosenberger 2018; Mokre 2018; Rosenberger/Hadj-Abdou 2013; Rosenberger/Haselbacher 2016; Rosenberger/Winkler 2013; 20I4; Ruedin et al. 2018). Viele AutorInnen sahen in diesem Zusammenhang die Stärke des Rechtspopulismus in der Wahlarena als Hauptgrund für die häufig als gering eingeschätzte Protestmobilisierung durch rechtsgerichtete AkteurInnen.

Auch die politische Dimension des Öffnungsprozesses, d. h. die europäische Integration, ist in Österreich ein stark von rechtspopulistischen Kräften politisiertes Thema, da ein relevanter euroskeptischer linker Gegenpol fehlt (Fallend 2008). Die Auswirkungen dieser Konfliktlinie auf die Protestpolitik fanden bislang jedoch nur wenig Beachtung (Dolezal et al. 2016a).

Die Forderungen der Umweltbewegung, der wichtigsten neuen sozialen Bewegung der I970er und I980er Jahre, lassen sich in einer von Auswirkungen der Globalisierung geprägten Konfliktstruktur nicht leicht verorten. Die starke Rolle der österreichischen Grünen hat die Aufmerksamkeit auf die Wahlarena gerichtet (Dolezal 20I6). Auf Basis von Befragungsdaten wurde in den 2oooer Jahren im Umweltbereich hingegen ein Rückgang der unkonventionellen Mobilisierung konstatiert (Hadler/Kaup 20II). Neben der bereits erwähnten AntiGMO-Bewegung (Seifert 2008; 2009) war in den letzten Jahren vor allem die Anti-AKW-Bewegung erneut ein Thema wissenschaftlicher Publikationen (Bayer 20I4; Thurner et al. 2017).

\subsection{Akteurskonfiguration}

Protestpolitik weist zahlreiche Querverbindungen sowohl zum Parteien- als auch zum Verbändesystem auf. Die relative Stärke (potenzieller) BündnispartnerInnen beeinflusst das Verhalten der HerausforderInnen, wobei die Literatur Unterschiede zwischen Links und Rechts betont: Die (neue) Linke gilt aufgrund ihrer anti-autoritären Orientierung als protestaffin, während rechte AkteurInnen in erster Linie auf institutionalisierte Kanäle setzen und Protestmobilisierung daher vor allem als Ausweichstrategie im Falle eines fehlenden Zugangs zu Parlamenten und Regierungen sehen (Hutter/Kriesi 20I3). Erst in jüngster Zeit kam es auch in Österreich bei rechten Gruppen wie den Identitären zu einer Übernahme von traditionell der Linken zugerechneten Aktionsformen (Bruns et al. 2016). Diesen Unterschied zeigt auch die Umfrageforschung, da sich trotz der generellen „Normalisierung“ (van Aelst/Walgrave 200I) linksori- entierte Personen immer noch stärker an Protesten beteiligen (Torcal et al. 2016).

Der hier behandelte Untersuchungszeitraum kann auf Basis der Zusammensetzung der Bundesregierung, des Machtzentrums der österreichischen Politik, in drei Abschnitte gegliedert werden: Während die Jahre 1998 und 1999 noch von der seit 1987 regierenden Großen Koalition aus SPÖ (Sozialdemokratische Partei Österreichs) und ÖVP (Österreichische Volkspartei) geprägt waren, führte die Bildung der ÖVP-FPÖ-Koalition im Februar 2000 zu einem deutlichen Einschnitt. Die Einbeziehung der rechtspopulistischen FPÖ (Freiheitliche Partei Österreichs) bzw., nach der 2005 erfolgten Parteispaltung, des BZÖ (Bündnis Zukunft Österreich) wurde auch international als Epochenbruch wahrgenommen. Dieser Abschnitt endete im Jänner 2007 mit der erneuten Bildung einer Großen Koalition, die erst 2017, d. h. nach Ende der hier untersuchten Periode, beendet wurde. Die Linke war demnach von 2000 bis Anfang 2007 mit einer Konstellation konfrontiert, in der sowohl die traditionelle (gemäßigte) Linkspartei SPÖ als auch die linksliberalen Grünen in Opposition waren. Die radikale Linke spielt in Österreichs Wahlarena, von einem lokalen Sonderfall abgesehen, keine Rolle. ${ }^{3}$

Die Machtverschiebungen zwischen den Parteien auf der Bundesebene widerspiegeln sich auch in der relativen Stärke der mit ihnen verbundenen Interessenorganisationen. Die Sozialpartnerschaft, so der einhellige Befund, hat seit längerem an Bedeutung verloren. Durch die Bildung der ÖVP-FPÖ-Regierung wurde sie weiter geschwächt und vor allem die Interessen der ArbeitnehmerInnen gerieten unter Druck (Karlhofer/Tálos 2019).

\subsection{Auswirkungen auf die Protestarena}

Welche Konsequenzen haben die eben skizzierten Entwicklungen des politischen Kontexts für die Protestarena? In Bezug auf die drei in weiterer Folge untersuchten Charakteristika - Mobilisierungsstärke, Aktionsformen und Themen - lassen sich die folgenden Erwartungshaltungen formulieren:

Im Hinblick auf das Ausmaß der Proteste ist aufgrund der spezifischen Ausgestaltung der Opportunitätsstrukturen eine allenfalls durchschnittliche Mobilisierung zu erwarten. Da ein Vergleich mit anderen Ländern nicht unternommen wird, richtet sich der Fokus jedoch auf die Entwicklung der Mobilisierungsstärke im untersuchten Zeitraum. Hier sind prägnante Veränderungen vor allem im Zusammenhang mit dem Wandel der Akteurskonfiguration zu erwarten, d. h. mit dem Regierungswechsel von 200o. Während der Eintritt der FPÖ (BZÖ) zu

3 Die Kommunistische Partei Österreichs (KPÖ) ist aufgrund ihrer Stärke in der Landeshauptstadt Graz seit 2005 wieder im steirischen Landtag vertreten (Weisskircher 2017). 
einer Protestmobilisierung von Links führen sollte, ist aufgrund der oben erwähnten Unterschiede zwischen linker und rechter Mobilisierung keine spiegelbildliche Entwicklung auf Seite der politischen Rechten zu erwarten, da die FPÖ (BZÖ) auch nach ihrem Ausscheiden aus der Regierung eine relevante Akteurin in der institutionalisierten Politik geblieben ist.

Die Akteurskonfiguration kann auch Auswirkungen auf die gewählten Aktionsformen haben. Wenn HerausforderInnen keine realistische Chance sehen, mit ihren Anliegen in das politische System integriert zu werden, greifen sie verstärkt auf härtere Protestformen zurück. Der spezifische, auf Inklusion abzielende Umgang mit HerausforderInnen ist jedoch ein Bestandteil der politischen Kultur und somit als ein längerfristig stabiles Phänomen zu sehen. Moderate Aktionsformen sollten daher weiterhin überwiegen.

Die Themen der Protestarena sollten den Wandel der generellen Konfliktlinien widerspiegeln, $d$. h. verstärkt von den neuen, mit der Öffnung von Nationalstaaten verbundenen Gegensätzen geprägt sein (vgl. Hutter 20I4). Kurzfristige Schwankungen sind - neben spezifischen Ereignissen - jedoch erneut von der Akteurskonfiguration beeinflusst. Gerade der als befriedet geltende traditionelle Klassenkonflikt kann bei einem sich ändernden Umfeld wieder an Schärfe gewinnen. Aus Sicht der oben erwähnten Movement Society-These (Meyer/ Tarrow 1998) ist schließlich ein breites Spektrum an Themen und Forderungen zu erwarten, da immer mehr AkteurInnen die Protestpolitik für ihre Anliegen nutzen.

\section{Methodik der Untersuchung}

Die Daten der vorliegenden Studie stammen aus einer für den Zeitraum von 1998 bis 2016 durchgeführten Protestereignisanalyse. Diese spezifische Form der quantitativen Inhaltsanalyse ist die beste Methode für die Untersuchung des Ausmaßes, vor allem aber der Charakteristika von politischem Protest sowohl über einen längeren Zeitraum hinweg als auch in einem größeren Territorium (Koopmans/Rucht 2002). Vor dem Beginn der Datensammlung müssen drei Entscheidungen getroffen werden. Diese beziehen sich auf die Definition von Protestereignissen, die Auswahl der Quellen und die gewählte Samplingstrategie (Hutter/Giugni 2009, 437). Die Studie folgt einer inklusiven, pragmatischen Definition von Protestereignissen, die alle beobachtbaren, mit politischem Protest verbundenen Verhaltensformen umfasst. Solche Formen „unkonventioneller" Partizipation umfassen unter anderem Unterschriftensammlungen/Petitionen, Demonstrationen, Boykotte, Streiks, Blockaden und Flash Mobs, aber auch illegale und gewalttätige Formen wie Sachbeschädigungen oder politisch motivierte Geiselnahmen. Rein verbale
Äußerungen oder Stellungnahmen in sozialen Medien werden nicht berücksichtigt. Welche Akteurstypen ein Protestereignis organisieren, ist nicht Gegenstand der Definition. Ferner wird - im Gegensatz zu anderen Studien $^{4}$ - keine Mindestgröße der Beteiligung definiert, da bestimmte Protestformen, wie z. B. Hungerstreiks, auch von Einzelpersonen durchgeführt werden. Auch die AdressatInnen des Protests werden als Variable, nicht als Definitionskriterium betrachtet und umfassen daher nicht nur staatliche AkteurInnen, sondern auch Firmen und öffentliche Organisationen, wie z. B. die katholische Kirche.

Die zweite Entscheidung, die Auswahl der Quelle, ist die wichtigste Weichenstellung. Lange Zeit nutzten Protestereignisanalysen zumeist Tageszeitungen, vor allem Qualitätsblätter (z. B. Dolezal/Hutter 2007; Kriesi et al. 1995). Die vorliegende Studie basiert im Gegensatz dazu auf Agenturmeldungen der APA. Diese Texte richten sich nicht an die breite Öffentlichkeit, bilden aber häufig die Basis für massenmediale Beiträge oder werden sogar wortwörtlich übernommen. Die APA, sie befindet sich im Eigentum von zwölf Tageszeitungen und des Österreichischen Rundfunks (ORF), ist der wichtigste Informationsdienst in Österreich. In vielen Bereichen kommt ihr eine Monopolstellung zu, weshalb JournalistInnen die APA als eine der wichtigsten Quellen für ihre Arbeit betrachten (Plasser/Lengauer 20IO, 84). Für die Protestforschung weisen Agenturmeldungen alle Vorteile von Artikeln in Tageszeitungen auf: Die Auswahl der Ereignisse erfolgt systematisch und kann mit der Nachrichtenwerttheorie (O`Neill/Harcup 2009) erklärt werden. Die Berichterstattung ist kontinuierlich und relativ genau. Und schließlich erleichtert die an Zeitungsartikel erinnernde Form der Darstellung die Aufnahme und Verarbeitung der Informationen bei der Datenerhebung (Earl et al. 2004; Rucht/Neidhardt 1998). Wie Zeitungsartikel sind APA-Meldungen elektronisch verfügbar, jedoch nicht über Universitätsbibliotheken oder andere öffentliche Einrichtungen. Sie müssen angekauft werden.

Das am häufigsten diskutierte Problem bei allen Formen von Mediendaten ${ }^{5}$ ist der Selektionsbias (Barranco/Wisler 1999; McCarthy et al. 1996). Das „Universum" der Proteste ist stets unbekannt, doch kann die Aufnahme eines Ereignisses in die Berichterstattung als Relevanzkriterium interpretiert werden. Koopmans und Rucht argumentieren sogar, dass Proteste, über die nicht berichtet wird, in gewisser Weise "nicht existieren“ (2002, 252). Auch die APA erfasst nicht jeden Protest in Österreich, doch berichtet sie weitaus ausführlicher als einzelne Medien. Ferner sind die EigentümerInnen der APA und damit die NutzerInnen der Meldungen über

4 PRODAT, ein deutsches Projekt, verwendet eine Mindestanzahl von drei Personen (Rucht et al. 1992, 6).

5 Polizeidaten, eine andere Quelle der Protestforschung, sind in Österreich nicht zugänglich. 
das gesamte Bundesgebiet verteilt, wogegen die meisten Tageszeitungen einen regionalen Schwerpunkt in ihrer Berichterstattung aufweisen. Und schließlich sind die EigentümerInnen, darunter etwa der liberale Standard oder die konservative Presse, politisch unterschiedlich ausgerichtet, weshalb die APA insgesamt ausgewogen berichtet.

In Bezug auf die dritte Entscheidung, die Samplingstrategie, basiert die vorliegende Analyse auf allen während des Untersuchungszeitraums veröffentlichten Agenturmeldungen. Da elektronische Archive den Selektionsprozess enorm erleichtern, ist es nicht mehr notwendig, die Analyse auf bestimmte Tage, etwa die Montagsausgaben von Tageszeitungen (z. B. Dolezal/Hutter 2007; Kriesi et al. 1995), zu beschränken. Dennoch ist der Arbeitsaufwand sehr hoch geblieben, da die notwendige Aufnahme verschiedener Suchwörter bei der (Volltext) Suche nach relevanten Artikeln zu einem sehr hohen Anteil falsch positiver Treffer führt. Für die vorliegende Untersuchung wurden mit einer langen Liste von Suchwörtern ${ }^{6}$ 199.33I Treffer aus einem Total von I.572.12I Meldungen über Ereignisse in Österreich erzielt. Zusätzlich wurden Jahresrückblicke der APA, d. h. kurze Zusammenfassungen von Schlüsselereignissen, die jeweils rund eine A4-Seite pro Monat und Ressort (z. B. Innenpolitik) umfassen, als Absicherung durchgesehen. ${ }^{7}$

Die Codierung der identifizierten Proteste umfasst unter anderem Angaben zu Ort, Zeitpunkt und Aktionsform des Ereignisses sowie die Anzahl der TeilnehmerInnen, für die, falls vorhanden, zwei Angaben (Minimum und Maximum) aufgenommen wurden. Ferner wurden vor allem die OrganisatorInnen und AdressatInnen der Proteste sowie die thematischen Forderungen sehr detailliert erfasst. Letzteres geschah mit einem System von rund 600 Kategorien, das im Rahmen der österreichischen nationalen Wahlstudie AUTNES (Austrian National Election Study) entwickelt wurde (Dolezal et al. 20I6b). ${ }^{8}$ Bis zu drei Issues konnten pro Protestereignis codiert werden, zusätzlich wurde die ideologische Ausrichtung der Forderung erfasst. Aufgrund der

6 Der Suchstring lautet: , (*aktionstag* OR *aktivist* OR *besetz* OR *blockade* OR *blockier* OR *boykott* OR *bürgerinitiative* OR *demo* OR *fackelz* OR *flash* OR * gewerkschaftlich* OR *kampfmaßnahme* OR *kampagne* OR *kundgebung* OR *lichterkette* OR *lichtermeer* OR *mahnwache* OR *marsch* OR *menschenkette* OR * petition* OR * protest* OR *streik* OR *transparent* OR *unterschrift* OR *unterstützungserklärung* OR *unterzeichne* OR *versammlung* ) nicht st=termine nicht ti=tagesprogramm nicht st=aviso nicht st=inlandspresse nicht st=kalenderblatt". Zusätzlich wurde die Sportberichterstattung aus der Analyse ausgeschlossen.

7 Die Selektion und Codierung der Meldungen erfolgten durch den Autor und vier studentische Hilfskräfte: Christina Auer, Paul Ali Majdzadeh-Ameli, Stefanie Rachbauer und Daniela Vukadin.

8 Diese Vorgehensweise weicht bewusst von der an der Bewegungsforschung orientierten Themencodierung bei Dolezal und Hutter (2007) ab. Die sehr feingliedrige Codierung ermöglicht jedoch direkte Vergleiche mit dem älteren Datensatz (vgl. Dolezal 2019a).
Unterschiede zur Wahlarena konnten während des Codierprozesses neue Themen vorgeschlagen werden, von denen schließlich 27 aufgenommen wurden. Sie erfassen unter anderem internationale Konflikte, die in österreichischen Wahlkämpfen keine Rolle spielen: etwa den Israel-Palästina-Konflikt.

\section{Ergebnisse der Analyse}

Mit Hilfe der Protestereignisanalyse können systematische Aussagen über die Entwicklung der österreichischen Protestarena im neuen Jahrtausend getroffen werden. Die Darstellung der Ergebnisse folgt den oben entwickelten Erwartungshaltungen und behandelt das Ausmaß der Proteste, deren Formen und die angesprochenen Themen.

Für die Mobilisierungsstärke können zwei Indikatoren herangezogen werden: die Anzahl der identifizierten Ereignisse und die Summe der dabei erfassten TeilnehmerInnen. ${ }^{9}$ Neben den über die Inhaltsanalyse der APA-Meldungen gewonnenen Daten präsentiert Abbildung 1 auch die Anzahl der TeilnehmerInnen bei Streiks, d. h. die Stärke der traditionellen ökonomischen Konfliktlinie. Die im Rahmen der Inhaltsanalyse selbst gewonnenen Daten zu Streiks und Betriebsversammlungen werden - wie in der gesamten vorliegenden Studie - nicht berücksichtigt.

Abbildung 1: Mobilisierungsstärke: Anzahl der Ereignisse und TeilnehmerInnen, 1998-2016

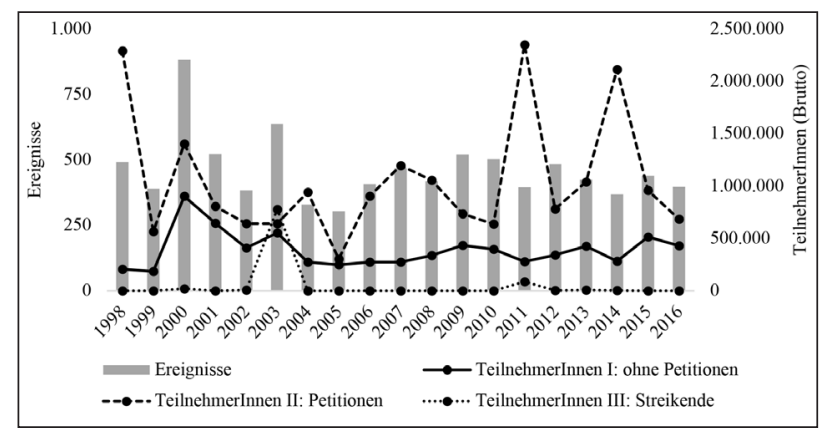

Quelle: eigene Erhebung (Ereignisse sowie TeilnehmerInnen I und II); ÖGB (TeilnehmerInnen III)

Abbildung 1 zeigt zunächst die mit Säulen ausgewiesene jährliche Anzahl der Proteste und dabei vor allem den hohen Wert von 882 erfassten Ereignissen im Jahr 2000, als die Bildung der ersten ÖVP-FPÖ-Regierung zur stärksten Protestbewegung seit 1945 führte. Zusätzlich gab es in diesem Jahr eine Welle von Protesten gegen Atomkraftwerke, bei denen AktivistInnen wiederholt

9 Bei bestimmten Aktionsformen, z. B. Besetzungen, ist auch die Ereignislänge ein relevanter Indikator. 
Tabelle 1: Aktionsformen der Proteste, 1998-2016 (Prozentwerte)

\begin{tabular}{lcccccc}
\hline & $1998-1999$ & $2000-2004$ & $2005-2009$ & $2010-2014$ & $2015-2016$ & Total \\
\hline Unterschriftensammlungen & 22,0 & 15,1 & 17,7 & 20,7 & 16,6 & 17,9 \\
demonstrative Formen & 66,3 & 68,4 & 63,3 & 64,7 & 65,8 & 65,8 \\
konfrontative Formen & 9,9 & 14,9 & 14,8 & 11,1 & 7,8 & 12,7 \\
leichte \& schwere Gewalt & 1,8 & 1,6 & 4,2 & 3,5 & 9,9 & 3,5 \\
(Anzahl) & $(882)$ & $(2756)$ & $(2121)$ & $(2741)$ & $(838)$ & $(8771)$ \\
\hline
\end{tabular}

Quelle: eigene Erhebung

Anmerkungen: „Demonstrative Formen“ sind u. a. Demonstrationen, Versammlungen und Mahnwachen, „konfrontative“ Besetzungen, Blockaden und Störungen von Kundgebungen. Alle Formen von Selbstbeschädigung (z. B. Hungerstreiks) werden als konfrontativ bewertet.

Grenzübergänge zu Tschechien blockierten. Ein weiterer Peak (N=638) folgt 2003, wofür auch Aktionen im Zusammenhang mit der großen Streikbewegung gegen eine Pensionsreform verantwortlich sind. Insgesamt wurden im Untersuchungszeitraum 8.77I Ereignisse erfasst, d. h. im Mittel 462 pro Jahr.

Die mit Linien dargestellten Zahlen der erfassten TeilnehmerInnen (sekundäre Y-Achse) sind der zweite Indikator für die Mobilisierungsstärke. Bei den Protestereignissen wird zwischen der Beteiligung bei Unterschriftensammlungen/Petitionen (TeilnehmerInnen II) und allen übrigen Aktionsformen (TeilnehmerInnen I) unterschieden. Insgesamt müssen diese Zahlen vorsichtig interpretiert werden, da der Anteil der codierten Ereignisse ohne Information zur Anzahl der TeilnehmerInnen mit 5I,6\% sehr hoch ausfällt. ${ }^{10} \mathrm{Gab}$ es für ein und dasselbe Protestereignis mehrere Angaben, typisch sind hier die "optimistischen" Schätzungen der VeranstalterInnen und die „konservativen“ Schätzungen der Polizei, wurde für die Analyse deren Mittelwert genommen. Solch widersprüchliche Angaben lagen in sechs Prozent der Fälle vor.

Auch die mit der durchgezogenen Linie erfasste Anzahl der TeilnehmerInnen I zeigt einen Höhepunkt im Jahr 2000, als sich insgesamt rund 900.000 Personen bei den erfassten Protesten beteiligten. Über den tatsächlichen Anteil an der Gesamtbevölkerung können solche Bruttoangaben jedoch keine Auskunft geben. Das jährliche Mittel der so erfassten Zahlen beträgt rund 400.00o. Deutlich mehr Personen beteiligen sich bei Unterschriftensammlungen, die mit der strichlierten Linie (TeilnehmerInnen II) ausgewiesen sind. Hierbei stechen vor allem drei Jahre heraus: 1998, 20II und 20I4: Neben mehreren umweltpolitischen Petitionen sammelte 1998 etwa die Katholische Arbeitnehmerbewegung Österreichs 269.000 Unterschriften für den arbeitsfreien Sonntag. Ferner wurden im Rahmen der internen Auseinandersetzungen um die Ausrichtung der katholischen Kirche 170.000 Unterschriften gegen

Io Auf eine Imputation fehlender Daten wird daher bewusst verzichtet. die Absetzung eines niederösterreichischen Pfarrers gesammelt. Die beiden anderen Spitzenjahre sind von jeweils einer sehr erfolgreichen Kampagne geprägt: 2OII übergaben TrafikantInnen rund I Mio. Unterschriften gegen eine Tabakprodukt-Richtlinie der EU; 2014 forderten ÖGB und Arbeiterkammer mit 882.I84 Unterschriften eine Lohnsteuersenkung.

Die mit der punktierten Linie ausgewiesene Entwicklung der Streikenden zeigt neben dem Spitzenwert von 2003 und einem deutlich kleineren Peak 20II sehr niedrige Werte. 2003 führten vor allem die Streiks gegen eine Pensionsreform zur stärksten Mobilisierung in der Geschichte der Zweiten Republik; 20II kam es zu Lohnkonflikten in der Metallindustrie. In nicht weniger als elf der 19 gezeigten Jahre gab es jedoch - auf Basis der vom ÖGB gebrauchten Definition ${ }^{\text {II }}$ - keine streikenden ArbeitnehmerInnen.

Die im Datensatz sehr detailliert erfassten Aktionsformen der Proteste, die zweite hier dargestellte Eigenschaft der österreichischen Protestarena, sind in Tabelle 1 in vier große Gruppen zusammengefasst. Die Einteilung der Zeiträume basiert auf adaptierten Jahrfünften, wobei die erste vollständige Periode im Jahr 2000 beginnt, um die Proteste gegen die ÖVP-FPÖ-Regierung in den frühen 20ooern erfassen zu können. Davon abgesehen dient die Periodisierung allein der Darstellung der allgemeinen Entwicklung des Protestgeschehens. Erst die letzte Periode (2015/2016) ist mit der Flüchtlingskrise wiederum stark von einem spezifischen Ereignis geprägt.

Bei der übergroßen Mehrheit der Protestereignisse handelt es sich um demonstrative Aktionsformen. Rund zwei Drittel aller Ereignisse fallen unter diese Kategorie, wozu vor allem Demonstrationen (Märsche) und Versammlungen (Kundgebungen), aber auch sehr viele kleine Proteste - etwa Mahnwachen - zählen. Im Zeitverlauf ist keine relevante Veränderung des Anteils dieser Formen zu erkennen. Bei den Unterschriftensamm-

II Diese Daten orientieren sich an spezifischen Eskalationsstufen und erfassen nicht alle streikähnlichen Proteste (z. B. Betriebsversammlungen mit Auswirkungen auf die Produktion bzw. den KundInnenverkehr). 
lungen gibt es ein Auf und $\mathrm{Ab}$, während konfrontative Protestformen, etwa Blockaden und Besetzungen, von der ersten zur zweiten bzw. dritten Untersuchungsperiode deutlich zunehmen. Dies ist zum Teil durch den Widerstand gegen die Bildung der ÖVP-FPÖ-Regierung verursacht, doch spielen hier auch die bereits erwähnten Proteste der Anti-AKW-Bewegung an Grenzübergängen (Blockaden) sowie die Proteste der Studierenden im Rahmen der unibrennt-Kampagne gegen das BolognaSystem (u. a. Besetzungen) eine Rolle. Bei der letzten angeführten Kategorie „leichte \& schwere Gewalt" zeigen die Daten eine Zunahme in der letzten Phase. Diese ist insgesamt stark von Protesten im Zusammenhang mit dem Thema Migration geprägt (siehe unten), wobei es auch zu Attacken auf Asylheime kam. Insgesamt liegt der Anteil dieser Kategorie über den gesamten Zeitraum hinweg jedoch unter vier Prozent und belegt die erwartete Dominanz gemäßigter Aktionsformen.

Bei den Themen der Proteste, dem dritten hier untersuchten Aspekt, sind unterschiedliche Analysestrategien möglich. Analog zur Wahlforschung kann etwa zwischen der Salienz (Bedeutung) und der Polarisierung von Themen, d. h. dem Ausmaß der unterschiedlichen Positionen, unterschieden werden. Die nachfolgende Darstellung beschränkt sich auf die Bedeutung der Themen, erfasst diese aber sowohl über die Anzahl der Ereignisse als auch die Summe der TeilnehmerInnen. Bei jedem Protestereignis konnten, wie erwähnt, bis zu drei (detaillierte) Themen erfasst werden, weshalb die Spaltensummen der in Tabelle 2 angeführten Prozentwerte stets größer als Ioo sind. Da bei den meisten Protesten sehr ähnliche Themen angesprochen werden und diese in der vorliegenden Auswertung häufig in dieselbe aggregierte Kategorie fallen, sind die Prozentsummen jedoch nicht besonders hoch. Die Reihenfolge der Themenkategorien orientiert sich an der Anzahl der über den gesamten Zeitraum erfassten Protestereignisse.

Auf Basis der hier verwendeten Kategorisierung ist Umweltschutz über den gesamten Untersuchungszeitraum hinweg das wichtigste Thema der österreichischen Protestarena. Mehr als ein Fünftel der Proteste kann diesem Politikfeld zugerechnet werden. Relevante Ereignisse waren etwa der Widerstand gegen den Einsatz von Gentechnik in der Landwirtschaft, Proteste gegen den (Aus-)Bau von Atomkraftwerken im benachbarten Ausland und viele Aktionen der Tierschutzbewegung. An zweiter Stelle folgt das hier als "Lebensstil“ bezeichnete Themenfeld, das der kulturellen Konfliktlinie zwischen libertären und autoritären Werten entspricht und - mit der wichtigen Ausnahme der Umwelt- sowie der Friedensbewegung (Kategorie: internationale Konflikte) - die Themen der neuen sozialen Bewegungen umfasst. Dieser Kategorie sind $u$. a. die Forderungen der Frauenund der LGBT-Bewegung sowie die Proteste ihrer GegnerInnen zugeordnet, aber auch das Thema Rechtsext- remismus, bei dem in den letzten Jahren vor allem die Proteste gegen Bälle von Burschenschaftern zu einer starken Mobilisierung geführt haben. Hinter diesen beiden Kategorien folgen die klassischen, der sozioökonomischen Konfliktlinie zuordenbaren Bereiche Wirtschaft (z. B. Lohnkonflikte oder das Thema Arbeitszeit) und Wohlfahrt (z. B. Pensionsreformen und Gesundheitspolitik). Ein näherer Blick auf die zeitliche Entwicklung der Daten zeigt erwartbare Ergebnisse, wie die große Bedeutung der Kategorie „Parteien \& Koalitionen“ in der ersten Hälfte der 20ooer Jahre oder die dominierende Position des Themas Migration am Ende der Untersuchung, d. h. in zeitlicher Nähe zur Flüchtlingskrise von 2015/2016. Hinter dem hohen Anteil der Kategorie "internationale Konflikte“ am Beginn der Untersuchung stehen u. a. Proteste im Zusammenhang mit der KosovoKrise und dem NATO-Militäreinsatz gegen Jugoslawien. Verglichen mit anderen Ländern spielte der Widerstand gegen den Irakkrieg hingegen eine deutlich geringere Rolle. Das Thema Bildung ist aufgrund der gewählten Periodisierung weniger gut sichtbar, da die letzte große Mobilisierungsphase, die unibrennt-Kampagne, in den Jahren 2009 und 2010 erfolgte.

Während die Themen Umwelt und „Lebensstil“ auf Basis der erfassten Ereignisse das Ranking anführen, sind es auf Basis der TeilnehmerInnen die Bereiche Wohlfahrt und Wirtschaft, da gerade in diesen Bereichen, wie weiter oben bereits erwähnt, einige größere Unterschriftensammlungen schlagend werden. Beim Thema Umwelt sind es im Gegensatz dazu häufig kleinere Proteste, etwa von TierschützerInnen. Sehr große, regelmäßig abgehaltene Protestereignisse im Bereich „Lebensstil“ sind jedoch z. B. die Regenbogenparaden der LGBT-Bewegung; dazu kommen noch die - deutlich kleineren - Gegenproteste.

Insgesamt kommt daher den Themen der neuen sozialen Bewegungen der I970er und I980er Jahre (Umweltschutz, "Lebensstil“), aber auch den klassischen soziökonomischen Feldern Wirtschaft und Wohlfahrt eine zentrale Rolle zu, wogegen die mit dem Öffnungsprozess von Nationalstaaten verbundenen Themen keineswegs dominieren: Nur I,4 Prozent der Proteste sprechen (auch) ökonomische Aspekte der Globalisierung an (z. B. Freihandelsabkommen). Migration, die kulturelle Dimension des Öffnungsprozesses, ist nur in der Endphase der vorliegenden Untersuchung zentral, wogegen Europa, die politische Dimension, generell keine Rolle in der Protestarena spielt. Die Daten deuten ein Mobilisierungspotenzial neuer und alter Gegensätze an, keinesfalls eine Dominanz der neuen, durch Globalisierungsprozesse ausgelösten Konfliktlinie.

Die Bandbreite der Themen ist im Zeitverlauf, wie von der Movement Society These erwartet, gewachsen: Ein direkter Vergleich der hier präsentierten Ergebnisse mit den älteren Daten von Dolezal und Hutter (2007) 


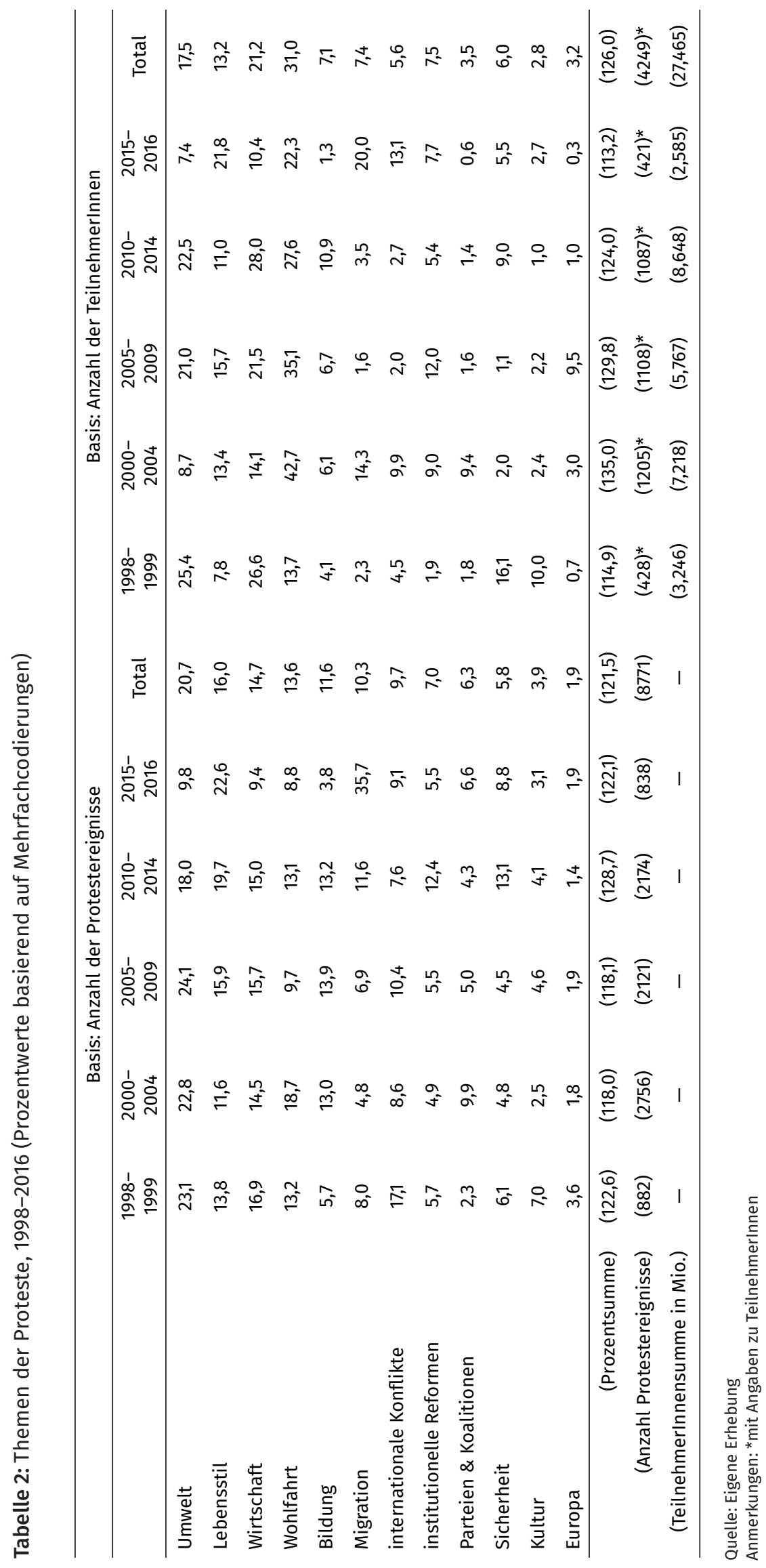


zeigt, dass die Variation der Themen von 1975 bis 2016 zugenommen hat (Dolezal 20I9a). In Bezug auf die hier nicht behandelte ideologische Ausrichtung der Proteste zeigen die Daten, dass im Normalfall nur eine Seite eines Konfliktes die Protestarena für ihre Anliegen nutzt: Proteste gegen Umweltmaßnahmen oder für Europa existieren, sind aber seltene Ereignisse. Allein der Themenbereich Migration führte zu einer Mobilisierung beider Seiten des Konfliktes - und das vor allem während der Flüchtlingskrise.

\section{Zusammenfassung}

Politischer Protest ist im 2I. Jahrhundert auch in Österreich ein alltägliches Element der politischen Auseinandersetzung geworden und hat daher seinen „unkonventionellen" Charakter endgültig verloren. Die "Normalität" von Protesten zeigt den Wandel von einer Konsens- zu einer Konfliktdemokratie, eine Entwicklung, die nicht allein mit Analysen der institutionalisierten Politik erfasst werden kann. Basierend auf einer systematischen Analyse der Berichterstattung der Nachrichtenagentur APA wurde daher ein umfassender Datensatz zu Protestereignissen erstellt, der im Moment den Zeitraum von 1998-20I6 abdeckt. Der vorliegende Beitrag folgte der theoretischen Konzeption des Politischen Prozess-Ansatzes und konzentrierte sich auf drei Charakteristika der österreichischen Protestarena: die Mobilisierungsstärke, die Aktionsformen und die Themen der Proteste.

Die Ergebnisse der Protestereignisanalyse zeigen, dass das im Jahr 2000 vor allem im Zusammenhang mit der Bildung der ersten ÖVP-FPÖ-Koalition erreichte Mobilisierungsniveau ein Ausreißer geblieben ist. Insgesamt zeigen die Daten zur Anzahl der identifizierten Ereignisse ein recht stabiles Bild. Im Gegensatz dazu ist die Anzahl der erfassten TeilnehmerInnen von größeren Schwankungen gekennzeichnet, wofür nicht zuletzt einzelne, sehr große Unterschriftensammlungen verantwortlich sind. Bei den Aktionsformen überwiegen gemäßigte Repertoires, erst in der letzten untersuchten Periode (2015-2016) ist es zu einem Anstieg bei der Gewalt gegen Sachen, aber auch Personen gekommen. Bei den Issues zeigt sich insgesamt eine Dominanz der Themen der neuen sozialen Bewegungen (Umweltschutz, „Lebensstil“ ), aber auch sozioökonomische Konfliktfelder (Wohlfahrt, Wirtschaft) haben ihr Mobilisierungspotenzial nicht eingebüßt. Im Vergleich dazu geraten die direkt mit der Öffnung von Nationalstaaten verbundenen Themen Europäische Integration und Migration in den Hintergrund. Letzteres dominierte jedoch die Protestarena in zeitlicher Nähe zur Flüchtlingskrise 2015/2016.

Analysen wie die vorliegende und der ihr zugrundeliegende Datensatz können aufgrund des Fokus auf ein einzelnes Land weniger gut den Einfluss von Kontextfaktoren auf der Makroebene testen, doch bieten sie im Gegensatz zu großen, vergleichenden Studien (zuletzt Kriesi et al. 2020) ein deutlich detaillierteres Bild des Geschehens. Verglichen mit Analysen einzelner Ereignisse oder AkteurInnen steht jede Protestereignisanalyse andererseits vor dem Problem, dass die zugrundeliegende Quelle niemals alle Informationen enthält. Allerdings ist es nur mit dieser Methode möglich, systematische Aussagen über die relative Bedeutung von AkteurInnen, Aktionsformen oder Themen zu treffen.

Der vorliegende Beitrag konzentrierte sich bewusst auf eine allgemeine Darstellung der Entwicklung des politischen Protests im untersuchten Zeitraum und ging dabei nur auf ausgewählte Aspekte ein. Nachfolgende Publikationen werden sich unter anderem mit ausgewählten Themen (z. B. Migration und Umweltschutz) und Akteurstypen (z. B. Parteien) beschäftigen. Ferner werden direkte Vergleiche mit der Themensalienz der Wahlarena und nicht zuletzt eine fortlaufende Aktualisierung des Datensatzes angestrebt.

\section{Literatur}

Ajanovic, Edma/Stefanie Mayer/Birgit Sauer (2016), Spaces of Right-Wing Populism and Anti-Muslim Racism in Austria. Identitarian Movement, Civil Initiatives and the Fight against 'Islamisation', in: Czech Journal of Political Science, Vol. 2(2), I3I-I48.

Atac, Ilker (2016), "Refugee Protest Camp Vienna": making citizens through locations of the protest movement, in: Citizenship Studies, Vol. 20(5), 629-646.

Badie, Bertrand/Pierre Birnbaum (1979), Sociologie de l'état, Paris: Grasset.

Barranco, José/Dominique Wisler (1999), Validity and systematicity of newspaper data in event analysis, in: European Sociological Review, Vol. I5(3), 30I-322.

Bayer, Florian (2014), Die Ablehnung der Kernenergie in Österreich: Ein Anti-Atom-Konsens als Errungenschaft einer sozialen Bewegung?, in: Momentum Quarterly. Zeitschrift für Sozialen Fortschritt, Vol. 3(3), I70-187.

Berntzen, Lars Erik/Manès Weisskircher (2016), Anti-Islamic PEGIDA Beyond Germany: Explaining Differences in Mobilisation, in: Journal of Intercultural Studies, Vol. 37(6), 556-573.

Boyle, Michael Shane (20II), The Criminalization of Dissent. Protest Violence, Activist Performance, and the Curious Case of the VolxTheaterKarawane in Genoa, in: TDR: The Drama Review, Vol. 55(4), II3-I27.

Brantner, Cornelia/Christian Schwarzenegger (2012), Der Fall unibrennt. Mobilisierung, Kommunikationsverhalten und kollektive Verständigung von Protest- 
und Kampagnengesellschaften heute, in: SWS-Rundschau, Vol. 52(3), 227-248.

Bruckmüller, Ernst (1996), Nation Österreich. Kulturelles Bewußtsein und gesellschaftlich-politische Prozesse, Wien: Böhlau.

Bruns, Julian/Kathrin Glösel/Natascha Strobl (2016), Die Identitären. Handbuch zur Jugendbewegung der Neuen Rechten in Europa, Münster: Unrast.

Caiani, Manuela/Paolo Graziano (2018), Europeanisation and social movements: the case of the Stop TTIP campaigns, in: European Journal of Political Research, Vol. 57(4), IO3I-IO55.

Dachs, Herbert/Peter Gerlich/Herbert Gottweis/Helmut Kramer/Volkmar Lauber/Wolfgang C. Müller/Emmerich Tálos (Hrsg.) (2006), Politik in Österreich. Das Handbuch, Wien: Manz.

De Moor, Joost/Michiel De Vydt/Katrin Uba/Mattias Wahlström (2020), New kids on the block: tacking stock of the recent cycle of climate activism, in: Social Movement Studies, online first.

Dolezal, Martin (2016), The Greens in Austria and Switzerland: Two successful opposition parties, in: Van Haute, Emilie (Hrsg.), Green Parties in Europe, Surrey: Ashgate, I5-4I.

Dolezal, Martin(2019a), From Party State to Movement Society? Conventional and Unconventional Democratic Practices in Austria, 1974-2018, in: Bischof, Günter/ David Wineroither (Hrsg.), Democracy in Austria, Innsbruck: innsbruck university press, I37-I55.

Dolezal, Martin (2019b), Libertäre und autoritäre Positionen im österreichischen Parteiensystem: Die Nationalratswahlkämpfe von 1945 bis 20I3, in: Dolezal, Martin/Peter Grand/Berthold Molden/David Schriffl (Hrsg.), Sehnsucht nach dem starken Mann? Autoritäre Tendenzen in Österreich seit 1945, Wien: Böhlau, II7-226.

Dolezal, Martin (2020), Zwischen Partizipation und Parteistrategie: Direkte Demokratie in Österreichs Bundesländern, in: Hermann, Andrea Tony/Daniela Ingruber/Flooh Perlot/Katrin Praprotnik/Christina Hainzl (Hrsg.), regional.national.föderal - Zur Beziehung politischer Ebenen in Österreich, Wien: Facultas, I59-I72.

Dolezal, Martin/Regina Becker/Swen Hutter (2016a), Protesting European integration: politicisation from below?, in: Hutter, Swen/Edgar Grande/Hanspeter Kriesi (Hrsg.), Politicising Europe: Integration and Mass Politics, Cambridge: Cambridge University Press, II2-I34.

Dolezal, Martin/Laurenz Ennser-Jedenastik/Wolfgang C. Müller/Anna Katharina Winkler (20I6b), Analyzing Manifestos in their Electoral Context: A New Approach Applied to Austria, 2002-2008, in: Political Science Research and Methods, Vol. 4(3), 64I-650.
Dolezal, Martin/Swen Hutter (2007), Konsensdemokratie unter Druck? Politischer Protest in Österreich, 1975-2005, in: Österreichische Zeitschrift für Politikwissenschaft, Vol. 36(3), 338-352.

Earl, Jennifer/Andrew Martin/John D. McCarthy/Sarah A. Soule (2004), The use of newspaper data in the study of collective action, in: Annual Review Of Sociology, Vol. 30, 65-80.

Eisinger, Peter K. (1973), The Conditions of Protest Behavior in American Cities, in: American Political Science Review, Vol. 67(I), II-28.

Fallend, Franz (2008), Euroscepticism in Austrian Political Parties: Ideologically Rooted or Strategically Motivated?, in: Szczerbiak, Aleks/Paul Taggart (Hrsg.), Opposing Europe? The Comparative Party Politics of Euroscepticism. Volume I. Case Studies and Country Surveys, Oxford: Oxford University Press, 2OI-220.

Giugni, Marco/Maria T. Grasso (Hrsg.) (2015), Austerity and Protest. Popular Contention in Times of Economic Crisis. Surrey: Ashgate.

Gottweis, Herbert (1997), Neue soziale Bewegungen in Österreich, in: Dachs, Herbert/Peter Gerlich/Herbert Gottweis/Franz Horner/Helmut Kramer/Volkmar Lauber/Wolfgang C. Müller/Emmerich Tálos (Hrsg.), Handbuch des politischen Systems Österreichs. Die Zweite Republik, Wien: Manz, 342-358.

Gruber, Oliver (2017), "Refugees (no Longer) Welcome“. Asylum Discourse and Policy in Austria in the Wake of the 2015 Refugee Crisis, in: Barlai, Melani/ Birte Fähnrich/Christina Griessler/Markus Rhomberger (Hrsg.), The Migrant Crisis: European Perspectives and National Discourses, Zürich: Lit-Verlag, 39-57.

Hadj Abdou, Leila/Sieglinde Rosenberger (2019a), Contesting the deportation state? Political change aspirations in protests against forced returns, in: Ethnic and Racial Studies, Vol. 42(16), IO2-II9.

Hadj Abdou, Leila/Sieglinde Rosenberger (2019b), Party activism: the permeability of the asylum protest arena in Austria, in: Social Movement Studies, Vol. I8(4), $391-407$.

Hadler, Markus/Gerd Kaup (2OII), Von schwindenden Protesten und couragierten Mülltrennerinnen. Umweltverhalten der ÖsterreicherInnen in den Jahren 1994/1995, 200I und 2010, in: SWS-Rundschau, Vol. 5I(3), 255-274.

Hafez, Farid (2016), Pegida in Parliament? Explaining the Failure of Pegida in Austria, in: German Politics and Society, Vol. 34(4), IOI-II8.

Haselbacher, Miriam/Sieglinde Rosenberger (2018), Protests Against the Reception of Asylum Seekers in Austria, in: Rosenberger, Sieglinde/Verena Stern/Nina Merhaut (Hrsg.), Protest Movements in Asylum and Deportation, Cham: Springer Open, 247-269. 
Helms, Ludger/David Wineroither (Hrsg.) (2012a), Die österreichische Demokratie im Vergleich, Baden-Baden: Nomos.

Helms, Ludger/David Wineroither (2012b), Nationalrat, Bundesregierung und Bundespräsident: Die gouvernementale Arena im internationalen Vergleich, in: Helms, Ludger/David Wineroither (Hrsg.), Die österreichische Demokratie im Vergleich, Baden-Baden: Nomos, I47-I70.

Hutter, Swen (2014), Protesting Culture and Economics in Western Europe. New Cleavages in Left and Right Politics, Minneapolis: University of Minnesota Press.

Hutter, Swen/Marco Giugni (2009), Protest Politics in a Changing Political Context: Switzerland, 1975-2005, in: Swiss Political Science Review, Vol. I5(3), 427-46I.

Hutter, Swen/Hanspeter Kriesi (2013), Movements of the Left, Movements of the Right Reconsidered, in: Stekelenburg, Jacquelien/Conny Roggeband/Bert Klandermans (Hrsg.), The Future of Social Movement Research. Dynamics, Mechanisms, and Processes, Minneapolis: University of Minnesota Press, 28I-298.

Karlhofer, Ferdinand/Emmerich Tálos (2019), Sozialpartnerschaft am Ende?, in: Tálos, Emmerich (Hrsg.), Die Schwarz-Blaue Wende in Österreich. Eine Bilanz, Wien: LIT Verlag, II5-I36.

Koopmans, Ruud/Dieter Rucht (2002), Protest Event Analysis, in: Klandermans, Bert/Suzanne Staggenborg (Hrsg.), Methods of Social Movement Research, Minneapolis: University of Minnesota Press, 23I259.

Kriesi, Hanspeter (2004), Political Context and Opportunity, in: Snow, David A./Sarah A. Soule/Hanspeter Kriesi (Hrsg.), The Blackwell Companion to Social Movements, Oxford: Blackwell Publishing, 67-90.

Kriesi, Hanspeter/Edgar Grande/Martin Dolezal/Marc Helbling/Dominic Höglinger/Swen Hutter/Bruno Wüest (20I2), Political Conflict in Western Europe, Cambridge: Cambridge University Press.

Kriesi, Hanspeter/Edgar Grande/Romain Lachat/Martin Dolezal/Simon Bornschier/Timotheos Frey (2008), West European Politics in the Age of Globalization, Cambridge: Cambridge University Press.

Kriesi, Hanspeter/Ruud Koopmans/Jan Willem Duyvendak/Marco G. Giugni (1995), New Social Movements in Western Europe. A Comparative Analysis, Minneapolis: University of Minnesota Press.

Kriesi, Hanspeter/Jasmine Lorenzini/Bruno Wüest/Silja Häusermann (Hrsg.) (2020), Contention in Times of Crisis. Recession and Political Protest in Thirty European Countries, Cambridge: Cambridge University Press.

Lehmbruch, Gerhard (1967), Proporzdemokratie. Politisches System und politische Kultur in der Schweiz und in Österreich, Tübingen: J. C. B. Mohr (Paul Siebeck).
Lijphart, Arend (2012), Patterns of Democracy. Government Forms and Performance in Thirty-Six Countries, New Haven: Yale University Press.

Luther, Kurt Richard (2015), The Primacy of Politics: Austria and the Not-so-Great Recession, in: Kriesi, Hanspeter/Takis S. Pappas (Hrsg.), European Populism in the Shadow of the Great Recession, Colchester: ECPR Press, I4I-I59.

Maireder, Axel/Christian Schwarzenegger (2012), A movement of connected individuals. Social media in the Austrian student protests 2009, in: Information, Communication \& Society, Vol. I5(2), I7I-I95.

McAdam, Doug (1982), Political Process and the Development of Black Insurgency, I930-I970, Chicago: The University of Chicago Press.

McCarthy, John D./Clark McPhail/Jackie Smith (1996), Images of Protest: Dimensions of Selection Bias in Media Coverage of Washington Demonstrations, 1982 and 199I, in: American Sociological Review, Vol. 6I(3), 478-499.

Meyer, Sarah/Sieglinde Rosenberger (2015), The Politicisation of Immigration in Austria, in: van der Brug, Wouter/Gianni D'Amato/Joost Berkhout/Didier Ruedin (Hrsg.), The Politicisation of Migration, London: Routledge, 3I-5I.

Meyer, David S./Sidney Tarrow (Hrsg) (1998), The Social Movement Society. Contentious Politics for a New Century. Lanham: Rowman \& Littlefield Publishers.

Mokre, Monika (2018), "We Demand Our Rights!" The Refugee Protest Camp Vienna, in: Rosenberger, Sieglinde/Verena Stern/Nina Merhaut (Hrsg.), Protest Movements in Asylum and Deportation, Cham: Springer Open, 205-22I.

Neidhardt, Friedhelm/Dieter Rucht (1993), Auf dem Weg in die „Bewegungsgesellschaft“? Über die Stabilisierbarkeit sozialer Bewegungen, in: Soziale Welt, Vol. 44(3), 305-326.

O'Neill, Deirdre/Tony Harcup (2009), News Values and Selectivity, in: Wahl-Jorgensen, Karin/Thomas Hanitzsch (Hrsg.), The Handbook of Journalism Studies, New York: Routledge, I6I-I74.

Plasser, Fritz/Günther Lengauer (2010), Politik vor Redaktionsschluss: Kommunikationsorientierungen von Macht- und Medieneliten in Österreich, in: Plasser, Fritz (Hrsg.), Politik in der Medienarena, Wien: Facultas, 53-IOO.

Plasser, Fritz/Gilg Seeber (2017), Politische Kultur und Demokratiebewusstsein in der Zweiten Republik im internationalen Vergleich, in: Helms, Ludger/David Wineroither (Hrsg.), Die österreichische Demokratie im Vergleich, Baden-Baden: Nomos, 337-364.

Plasser, Fritz/Gilg Seeber/Peter A. Ulram (2000), Breaking the Mold: Politische Wettbewerbsräume und Wahlverhalten Ende der neunziger Jahre, in: Plasser, Fritz/ 
Peter Ulram/Franz Sommer (Hrsg.), Das österreichische Wahlverhalten, Wien: Signum, 55-II5.

Plasser, Fritz/Franz Sommer (2018), Wahlen im Schatten der Flüchtlingskrise. Parteien, Wähler und Koalitionen im Umbruch, Wien: Facultas.

Plasser, Fritz/Peter A. Ulram (2002), Das österreichische Politikverständnis. Von der Konsens zur Konfliktkultur?, Wien: WUV-Universitätsverlag.

Rone, Julia (2020), Contesting Austerity and Free Trade in the EU. Protest Diffusion in Complex Media and Political Arenas, Abgindon: Routledge.

Rosenberger, Sieglinde (20I2), Inklusive Demokratie? Die politischen, sozialen und religiösen Rechte von Wohnbürgern ohne österreichische Staatsbürgerschaft, in: Helms, Ludger/David Wineroither (Hrsg.), Die österreichische Demokratie im Vergleich, BadenBaden: Nomos, 383-40I.

Rosenberger, Sieglinde/Leila Hadj-Abdou (2013), Islam at issue. Anti-Islamic mobilization of the extreme right in Austria, in: Mammone, Andrea/Emmanuel Godin/ Brian Jenkins (Hrsg.), Varieties of Right-Wing Extremism in Europe, London: Routledge, I49-I63.

Rosenberger, Sieglinde/Miriam Haselbacher (2016), Populistischer Protest. Mobilisierung gegen Asylunterkünfte in oberösterreichischen Gemeinden, in: SWSRundschau, Vol. 56(3), 399-42I.

Rosenberger, Sieglinde/Gilg Seeber (2007), Zivilgesellschaft durch Direkte Demokratie? Volksbegehren in Österreich, in: Der Bürger im Staat, Vol. 57(4), 233-239.

Rosenberger, Sieglinde/Jakob Winkler (2013), Anti-Abschiebungsproteste: Mit Empathie gegen Exklusion, in: Atac, Ilker/Sieglinde Rosenberger (Hrsg.), Politik der Inklusion und Exklusion, Göttingen: V\&R unipress, III-I34.

Rosenberger, Sieglinde/Jakob Winkler (2014), Com/passionate Protests: Fighting the Deportation of Asylum Seekers, in: Mobilization, Vol. I9(2), I65-I84.

Rucht, Dieter (2007), The Spread of Protest Politics, in: Dalton, Russell J./Hans-Dieter Klingemann (Hrsg.), The Oxford Handbook of Political Behavior, Oxford: Oxford University Press, 708-723.

Rucht, Dieter/Peter Hocke/Thomas Ohlemacher (1992), Dokumentation und Analyse von Protestereignissen in der Bundesrepublik Deutschland (Prodat) Codebuch, Berlin: Wissenschaftszentrum für Sozialforschung (WZB).

Rucht, Dieter/Friedhelm Neidhardt (1998), Methodological Issues in Collecting Protest Event Data: Units of Analysis, Sources and Sampling, Coding Problems, in: Rucht, Dieter/Ruud Koopmans/Friedhelm Neidhardt (Hrsg.), Acts of Dissent. New Developments in the Study of Protest, Berlin: Edition Sigma, 65-89.

Ruedin, Didier/Sieglinde Rosenberger/Nina Merhaut (2018), Tracing Anti-deportation Protests: A Longitudinal Comparison of Austria, Germany and Switzerland, in: Rosenberger, Sieglinde/Verena Stern/Nina Merhaut (Hrsg.), Protest Movements in Asylum and Deportation, Cham: Springer Open, 89-II5.

Seifert, Franz (2008), Einhellige Abschottung und globalisierungskritische Bauernrevolte - Widerstand gegen die grüne Gentechnik in Österreich und Frankreich, in: SWS-Rundschau, Vol. 48(4), 485-504.

Seifert, Franz (2009), Consensual NIMBYs, Contentious NIABYs: Explaining Contrasting Forms of Farmers GMO Opposition in Austria and France, in: Sociologia Ruralis, Vol. 49(I), 20-40.

Tarrow, Sidney (1998), Power in Movement. Social Movements and Contentious Politics, Cambridge: Cambridge University Press.

Thurner, Paul W./Sylvain Brouard/Martin Dolezal/Isabel Guineaudau/Swen Hutter/Wolfgang C. Müller (20I7), The Conflict over Nuclear Energy: Public Opinion, Protest Movements, and Green Parties in Comparative Perspective, in: Müller, Wolfgang C./Paul W. Thurner (Hrsg.), The Politics of Nuclear Energy in Western Europe, Oxford: Oxford University Press, 65-97.

Torcal, Mariano/Toni Rodon/Maria José Hierro (2016), Word on the Street: The Persistence of Leftist-Dominated Protest in Europe, in: West European Politics, Vol. 39(2), 326-350.

van Aelst, Peter/Stefaan Walgrave (200I), Who is that (wo) man in the street? From the normalisation of protest to the normalisation of the protester, in: European Journal of Political Research, Vol. 39(4), 46I-486.

Walgrave, Stefaan/Dieter Rucht (Hrsg.) (20IO), The World Says No to War: Demonstrations against the War on Iraq, Minneapolis: University of Minnesota Press.

Weisskircher, Manès (2017), The Electoral Success of the Radical Left: Explaining the Least Likely Case of the Communist Party in Graz, in: Government and Opposition, Vol. 54(I), I45-I66.

\section{Autor}

Martin Dolezal ist Senior Scientist am Fachbereich Politikwissenschaft und Soziologie (Abteilung Politikwissenschaft) der Universität Salzburg und Fellow am Institut für Höhere Studien (IHS) in Wien. In seiner Forschung befasst sich Dolezal vor allem mit Fragen der politischen Partizipation und des Parteienwettbewerbs. 